\title{
Backscattering Improvement of UHF RFID Tag Efficiency
}

\author{
Aggelos Bletsas $^{(1)}$, Antonis G. Dimitriou ${ }^{(2)}$, and John N. Sahalos ${ }^{(3)}$ \\ (1) Technical University of Crete, Department of Electronic \& Computer Engineering, Akrotiri Campus \\ 73100 Chania, Crete, Greece \\ Email:aggelos@telecom.tuc.gr \\ (2,3)Aristotle University of Thessaloniki,Department of Electrical \& Computer Engineering ${ }^{(2)}$, Department of \\ Physics ${ }^{(3)}$, Thessaloniki, Greece \\ Email:antodimi ${ }^{(2)} /$ sahalos $^{(3)} @$ auth.gr
}

\begin{abstract}
In this work, a tag-load selection methodology is proposed for optimized tag-to-reader backscatter communication. Derivation of the method is based on antenna/communication theory and applies to any tag-antenna, including minimum scattering antennas as a special case. In contrast to what is commonly believed, it is shown that amplitude maximization of complex reflection coefficient difference between the two states is not a sufficient condition for optimized tag's design. Maximization of backscatter carrier power per bit must be sought as well. Optimum loadselection for passive and semi-passive tags is linked to the tag-antenna's structural mode. A method that allows for the closed-form calculation of this parameter is put forward.
\end{abstract}

\section{INTRODUCTION}

Numerous applications of RF/RFID systems have emerged in diverse fields, like aerospace, healthcare, logistics, supply chain management, and defense [1]. A tag modulates the signal transmitted by the reader, by connecting alternatively to two different loads, as demonstrated in Fig. 1. In our work, it is shown that:

- For optimizing the backscatter communication; except of the amplitude reflection coefficient difference, tag antenna structural mode must be considered.

- For the radar cross section (RCS) derivation of the tag antenna, a closed-form expression of the appropriate structural mode is needed and will be given.

The proposed tag-load selection applies to any tag antenna, including minimum scattering antennas as a special case, and covers both passive as well as semi-passive tags.

\section{CONNECTION TO PRIOR ART}

The RCS of the tag's antenna depends on the connected load. Let $\vec{E}_{i}$ denote the complex backscattered field from the tag connected to a load $Z_{i}\left(i=1\right.$ for bit ' 0 ' or $i=2$ for bit ' 1 '). The tag's $\operatorname{RCS} \sigma_{i}$ in the two states is then given as, [2]:

$$
\sigma_{i} \stackrel{\Delta}{=} \lim _{r \rightarrow+\infty} 4 \pi r^{2} \frac{\left|\vec{E}_{i}\right|^{2}}{\left|\vec{E}_{\text {ind }}\right|^{2}}=\frac{\lambda^{2}}{4 \pi} G^{2}\left|\Gamma_{i}-A_{s}\right|^{2},
$$

$A_{s}$ corresponds to the structural mode of the antenna, $G$ is the antenna's gain, $\lambda$ the wavelength, $\vec{E}_{\text {ind }}$ the induced field at the tag, and $\Gamma_{i}$ is the load-dependent reflection coefficient of the tag antenna-load system given by:

$$
\Gamma_{i} \stackrel{\Delta}{=} \frac{Z_{i}-Z_{a}^{*}}{Z_{i}+Z_{a}}
$$

$Z_{a}$ is that tag antenna's impedance.

The authors in [3] exploited the Thevenin equivalent circuit of an antenna, carefully noting that such modeling assumes minimum scattering antennas. In such a case, [3]-[4], RCS equals to:

$$
\sigma_{i}^{\text {thev }}=\frac{\lambda^{2} G^{2} R_{a}^{2}}{\pi\left|Z_{i}+Z_{a}\right|^{2}},
$$

Then, [3], they denote differential backscattered power as $P_{\text {diff.bs }}=1 / 2\left|I_{1}-I_{2}\right|^{2} R_{a} G$, where $I_{i}$ is the current flowing at the Thevenin circuit corresponding to the two loads $Z_{i}$ and define differential RCS $\Delta \sigma=P_{\text {diff.bs }} / S$, where $S$ is the induced power density (in $\mathrm{W} / \mathrm{m}^{2}$ ) at the tag location from the field transmitted by the reader. Finally, they show that: 


$$
\Delta \sigma=\frac{\lambda^{2} G^{2}}{4 \pi}\left|\Gamma_{1}-\Gamma_{2}\right|^{2}
$$

Therefore, the system designer should maximize $\left|\Gamma_{1}-\Gamma_{2}\right|$, when the tag is connected to a minimum scattering antenna. $1 / 2|I|^{2} R_{a} G$ is a valid representation of the backscattered power from a tag, only for minimum scattering antennas. For example, for an open circuit load, thus $Z_{i}=\infty$, by substituting in (3), the back-scattered RCS, based on the Thevenin equivalent circuit, is $\sigma_{i}^{\text {thev }}=0$. On the contrary, by substituting in (1), which models the general tag-antenna case, $\sigma\left(Z_{i}=\infty\right)=\left(\lambda^{2} / 4 \pi\right) G^{2}\left|1-A_{s}\right|^{2} \neq 0$. The latter equals 0 only when $A_{s}=1$. As shown in [5], the antenna structural mode is generally different than unity.

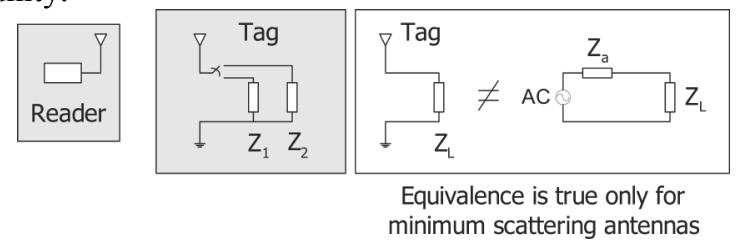

Fig. 1 Thevenin equivalent circuit for tag antennas.

\section{TAG EFFICIENCY CONSTRAINTS}

The scattered field $\vec{E}_{i}$ at location $(r, \theta, \varphi)$ when tag antenna is loaded with $Z_{i}$ can be expressed as [6], [7]:

$$
\vec{E}_{i} \equiv \vec{E}_{i}(r, \theta, \varphi)=\underbrace{I_{\text {ind }} \frac{\vec{E}_{a}(r, \theta, \varphi)}{I_{a}}}_{\vec{E}_{0}}\left(A_{s}-\Gamma_{i}\right)=\vec{E}_{0}(r, \theta, \varphi)\left(A_{s}-\Gamma_{i}\right)=\vec{E}_{0}\left(A_{s}-\Gamma_{i}\right)
$$

$I_{\text {ind }}$ is the induced current by the incoming field at the tag antenna terminals, when the tag is terminated at load $Z_{a}^{*}$. $\vec{E}_{a}$ is the field radiated by the tag antenna at location $(r, \theta, \varphi)$, when the current at the tag antenna terminals is $I_{a}$ and no external incident wave is applied to the tag antenna. The term $\vec{E}_{0}$ is independent of the tag load $Z_{i}$. By definition, the power $P_{i}$ of the backscattered field for the two terminating loads $Z_{i}(i=1,2)$ is given as:

$$
P_{i}=(1 / 2 \eta)\left|\vec{E}_{i}\right|^{2}=S \sigma_{i}
$$

From (6), it must be pointed out that the backscattered signal from the same tag and the same propagation conditions is not constant but depends on the tag's load, that is selected based on the tag bit information ( $S \sigma_{1}$ for bit ' 0 ' or $S \sigma_{2}$ for bit ' 1 '). Therefore, from (5), backscattered power depends on the complex reflection coefficient $\Gamma_{i}$, which is shaped by the tag's load as given in (2).

\section{A. Constraint 1. Maximize Backscattered Carrier Power per Bit.}

System designers should select loads $Z_{i}$, so that the average backscattered carrier power per bit $\left(P_{1}+P_{2}\right) / 2$ is maximized, or equivalently, by replacing in (6):

$$
\max \left\{\sigma_{1}+\sigma_{2}\right\}
$$

In (1) it was shown that $\sigma_{i}$ depends both on the load as well as on the load-independent antenna's structural mode $A_{s}$. So, it is important to derive the structural mode.

\section{B. Constraint 2. Minimize Bit-Error-Rate Probability (BER) at the Reader}

The tag designer should also select terminating loads $Z_{i}$ at the tag, such that BER at the reader is minimized. The signal $y_{i}$ received at the reader antenna is directly proportional to the backscattered field $\vec{E}_{i}$, given by (5), plus the additive receiver thermal noise:

$$
y_{i}=\underbrace{a \vec{E}_{i}}_{\mathrm{x}_{i}}+n,
$$

$a$ accounts for the transform of a field quantity (e.g. in $\mathrm{V} / \mathrm{m}$ ) to a signal quantity (e.g. in V). It is further assumed that thermal noise $n$ is a complex, zero-mean, circularly symmetric Gaussian random variable with expected power $E\left\{|n|^{2}\right\}=N_{0}$. Assuming knowledge of $a$, maximum likelihood (ML) detection of $\mathrm{x}_{1}=a \vec{E}_{1}$ for bit '0' or $\mathrm{x}_{2}=a \vec{E}_{2}$ for bit ' 1 ', in the presence of zero-mean, additive complex circularly symmetric gaussian noise, amounts to selecting bit ' 0 ' 
when the received signal $y$ is closer to $x_{1}\left(\left|y-x_{1}\right|<\left|y-x_{2}\right|\right)$, or bit '1' when the received signal is closer to $x_{2}$ $\left(\left|\mathrm{y}-\mathrm{x}_{1}\right|>\left|\mathrm{y}-\mathrm{x}_{2}\right|\right)$. In other words, detection error $e$ is performed when the amount of noise exceeds half the distance between $x_{1}$ and $x_{2}$. The probability of such event can be directly computed (e.g. see appendix in [8]), providing an expression of the BER at the reader

$$
\operatorname{Pr}\{e\}^{\Delta}=Q\left(\frac{\left|\mathrm{x}_{1}-\mathrm{x}_{2}\right|}{2 \sqrt{N_{0} / 2}}\right)=Q\left(\left|a \vec{E}_{0}\right| \frac{\left|A_{s}-\Gamma_{1}-A_{s}+\Gamma_{2}\right|}{2 \sqrt{N_{0} / 2}}\right)=Q\left(\left|a \vec{E}_{0}\right| \frac{\left|\Gamma_{1}-\Gamma_{2}\right|}{2 \sqrt{N_{0} / 2}}\right)
$$

where $Q(x)=(1 / \sqrt{2 \pi}) \int_{x}^{+\infty} \exp \left(-x^{2} / 2\right) d x$ is the Q-function [9], which decreases with increasing $x$. Therefore, BER minimization requires maximization of the reflection coefficient difference amplitude:

$$
\max \left\{\left|\Gamma_{1}-\Gamma_{2}\right|\right\}
$$

Notice that the above derivation has employed simple detection theory, without any type of Thevenin-based tag antenna-tag chip modeling or any prior assumption regarding the tag antenna or the tag circuitry. Also, it applies to any binary modulation. The result of (9) agrees with [3], where Thevenin equivalent circuit and minimum scattering antennas were considered. Moreover, from (9), maximization of the load-independent antenna-specific term $\left|\vec{E}_{0}\right|$ is also needed.

\section{CLOSED-FORM CALCULATION OF TAG ANTENNA STRUCTURAL MODE}

In order to calculate the structural mode parameter $A_{s}$, three values $\sigma_{1}, \sigma_{2}, \sigma_{3}$ of the antenna RCS are needed, corresponding to three different loads $Z_{1}, Z_{2}, Z_{3}$ (or equivalently reflection coefficients $\Gamma_{1}, \Gamma_{2}, \Gamma_{3}$ ). Antenna RCS can be measured experimentally [5] or estimated through simulation. Denote complex $\Gamma_{i}=x_{i}+j y_{i}$ that corresponds to load $Z_{i}$ and RCS $\sigma_{i}$, with $i \in\{1,2,3\}$ and the unknown $A_{s}=x+j y$. From (1), we have three circle equations $\left(\mathbf{c}_{1}, \mathbf{c}_{2}, \mathbf{c}_{3}\right)$ :

$$
\left(x-x_{1}\right)^{2}+\left(y-y_{1}\right)^{2}=\frac{4 \pi}{\lambda^{2} G^{2}} \sigma_{1},\left(\mathbf{c}_{1}\right),\left(x-x_{2}\right)^{2}+\left(y-y_{2}\right)^{2}=\frac{4 \pi}{\lambda^{2} G^{2}} \sigma_{2},\left(\mathbf{c}_{2}\right),\left(x-x_{3}\right)^{2}+\left(y-y_{3}\right)^{2}=\frac{4 \pi}{\lambda^{2} G^{2}} \sigma_{3},\left(\mathbf{c}_{3}\right)
$$

Dividing $\left(\mathbf{c}_{1}\right)$ with $\left(\mathbf{c}_{2}\right)$ and setting $k_{12}=\sigma_{l} / \sigma_{2}$, we get a new circle centered at $\left(x_{1}^{*}, y_{1}^{*}\right)$, with radius $r_{1}$ :

$$
\begin{aligned}
& \left(x-x_{1}^{*}\right)^{2}+\left(y-y_{1}^{*}\right)^{2}=r_{1}^{2}, \\
& \left(x_{1}^{*}, y_{1}^{*}\right)=\left(\frac{x_{1}-\kappa_{12} x_{2}}{1-\kappa_{12}}, \frac{y_{1}-\kappa_{12} y_{2}}{1-\kappa_{12}}\right), r_{1}^{2}=\left(\frac{x_{1}-\kappa_{12} x_{2}}{1-\kappa_{12}}\right)^{2}+\left(\frac{y_{1}-\kappa_{12} y_{2}}{1-\kappa_{12}}\right)^{2}+\frac{\kappa_{12}\left(x_{2}^{2}+y_{2}^{2}\right)}{1-\kappa_{12}}-\frac{x_{1}^{2}+y_{1}^{2}}{1-\kappa_{12}}
\end{aligned}
$$

Similarly, by dividing $\left(\mathbf{c}_{1}\right)$ with $\left(\mathbf{c}_{3}\right)$ and setting $k_{13}=\sigma_{1} / \sigma_{3}$, we get a circle centered at $\left(x_{2}^{*}, y_{2}^{*}\right)$, with radius $r_{2}$

$$
\begin{aligned}
& \left(x-x_{2}^{*}\right)^{2}+\left(y-y_{2}^{*}\right)^{2}=r_{2}^{2}, \\
& \left(x_{2}^{*}, y_{2}^{*}\right)=\left(\frac{x_{1}-\kappa_{13} x_{3}}{1-\kappa_{13}}, \frac{y_{1}-\kappa_{13} y_{3}}{1-\kappa_{13}}\right), r_{2}^{2}=\left(\frac{x_{1}-\kappa_{13} x_{3}}{1-\kappa_{13}}\right)^{2}+\left(\frac{y_{1}-\kappa_{13} y_{3}}{1-\kappa_{13}}\right)^{2}+\frac{\kappa_{13}\left(x_{3}^{2}+y_{3}^{2}\right)}{1-\kappa_{13}}-\frac{x_{1}^{2}+y_{1}^{2}}{1-\kappa_{13}}
\end{aligned}
$$

Finally, dividing $\left(\mathbf{c}_{2}\right)$ with $\left(\mathbf{c}_{3}\right)$ and setting $k_{23}=\sigma_{2} / \sigma_{3}$, we get a circle centered at $\left(x_{3}^{*}, y_{3}^{*}\right)$, with radius $r_{3}$

$$
\begin{aligned}
& \left(x-x_{3}^{*}\right)^{2}+\left(y-y_{3}^{*}\right)^{2}=r_{3}^{2}, \\
& \left(x_{3}^{*}, y_{3}^{*}\right)=\left(\frac{x_{2}-\kappa_{23} x_{3}}{1-\kappa_{23}}, \frac{y_{2}-\kappa_{23} y_{3}}{1-\kappa_{23}}\right), r_{3}^{2}=\left(\frac{x_{2}-\kappa_{23} x_{3}}{1-\kappa_{23}}\right)^{2}+\left(\frac{y_{2}-\kappa_{23} y_{3}}{1-\kappa_{23}}\right)^{2}+\frac{\kappa_{23}\left(x_{3}^{2}+y_{3}^{2}\right)}{1-\kappa_{23}}-\frac{x_{2}^{2}+y_{2}^{2}}{1-\kappa_{23}}
\end{aligned}
$$

The intersection of circles (11) and (12) gives:

$$
\begin{aligned}
& (x, y)=\left(x_{1}^{*}+\frac{a}{d}\left(x_{2}^{*}-x_{1}^{*}\right) \pm \frac{h}{d}\left(y_{2}^{*}-y_{1}^{*}\right), y_{1}^{*}+\frac{a}{d}\left(y_{2}^{*}-y_{1}^{*}\right) \mp \frac{h}{d}\left(x_{2}^{*}-x_{1}^{*}\right)\right), \\
& \text { where } d=\sqrt{\left(x_{1}^{*}-x_{2}^{*}\right)^{2}+\left(y_{1}^{*}-y_{2}^{*}\right)^{2}}, a=\frac{r_{1}^{2}-r_{2}^{2}+d^{2}}{2 d}, h=\sqrt{r_{1}^{2}-a^{2}}
\end{aligned}
$$

The pair $(x, y)$ above that validates (13) provides the unknown $A_{s}=x+j y$. As an example, consider the case of $Z_{a}=6.7+148.8 j, \Gamma_{1}=1$ (open circuit) with $\sigma_{1}=0.0098 \mathrm{~m}^{2}, \Gamma_{2}=0$ (matched load) with $\sigma_{2}=0.0148 \mathrm{~m}^{2}$ and $\Gamma_{3}=j$ (reactive load) with $\sigma_{3}=0.0146 \mathrm{~m}^{2}$ [5]. The result of the above calculation provides exact value of $A_{s}=0.6047+0.5042 j$, which is close to the approximate value provided in [5], where $A_{s}$ is graphically estimated on a Smith chart. 


\section{EFFICIENT TAGS: CASE STUDY - DISCUSSION}

In the next paragraphs, the proposed methodology is applied in the design of a passive and a semi-passive tag. Loads are selected, with respect to the tag-antenna's structural mode, for optimized backscatter communication.

\section{A. Case I: Passive Tags}

For battery-less passive tags, tag load $Z_{1}$ usually corresponds to perfect match $\left(\Gamma_{1}=0\right)$. In that way, power transfer from tag antenna to tag chip is maintained, at least for the duration of bit ' 0 '. The tag load $Z_{2}$ (and corresponding $\Gamma_{2}$ ) is selected such that $\Gamma_{2}-A_{s}$ is collinear with $\Gamma_{1}-A_{s}$. Two possible solutions for $\Gamma_{2}$ on the unit circle are depicted: solution I and II (Fig. 2-left). Both satisfy constraint 2 given in (9), while solution II provides for higher average backscatter carrier power per bit (constraint 1) than solution I, since $\left|\Gamma_{2}-A_{s}\right|$ is maximized. For the special case of $A_{s}=1$, one solution could be $\Gamma_{2}=A_{s}=1$. A better solution with higher backscattered carrier power per bit would be $\Gamma_{2}=-1$.

\section{B. Case II: Semi-Passive Tags}

Given that power transfer from reader (or tag antenna) to the tag chip is not needed during tag-to-reader communication, $\Gamma_{1}$ need not be equal to zero, while both reflection coefficients should be on the unit circle (in order to maximize $\left|\Gamma_{1}-\Gamma_{2}\right|$ ). Fig. 2-right depicts two solutions that both achieve the maximum $\left|\Gamma_{1}-\Gamma_{2}\right|=2$. In general, solution II could represent any two diametrically opposite points on the unit circle. Both Solutions I and II in Fig. 2right, as well as any two diametrically opposite points on the unit circle achieve the same $\sigma_{1}+\sigma_{2}$ (constraint 1 of (7)). The proof follows: denote $\Gamma_{1}=a+j b$, diametrically opposite $\Gamma_{2}=-a-j b$, both on the unit circle $|a|^{2}+|b|^{2}=1$ and $A_{s}=x+j y$ :

$$
\sigma_{1}+\sigma_{2} \propto\left|\Gamma_{1}-A_{s}\right|^{2}+\left|\Gamma_{2}-A_{s}\right|^{2}=|(a-x)+j(b-y)|^{2}+|-(a+x)-j(b+y)|^{2}=2+2\left|A_{s}\right|^{2}
$$

In other words, any diametrically opposite $\left(\Gamma_{1}, \Gamma_{2}\right)$ on the unit circle satisfy both constraints. However, by selecting $\Gamma_{\mathrm{i}}$ on the line segment that is vertical to $\mathrm{OA}_{\mathrm{s}}$ (Fig. 2-right) and crosses $\mathrm{O}$, back-scattered power given by (5), (6) is equal for the two loads, achieving zero-tag efficiency variance.


Fig. 2 Tag load selection and corresponding reflection coefficients $\Gamma_{i}$ for a passive and a semi-passive tag.

This work is co-funded by the European Union and the Research Promotion Foundation of Cyprus.

\section{REFERENCES}

[1] D. M. Dobkin, The RF in RFID: Passive UHF RFID in Practice, Newnes (Elsevier), 2008.

[2] C. A. Balanis, Antenna Theory: Analysis and Design, $3^{\text {rd }}$ ed., John Wiley and Sons, New Jersey, 2005.

[3] P. V. Nikitin, K. V. S. Rao, and R. Martinez, "Differential RCS of RFID tag," Electronics Letters, vol. 43, no. 8, pp. 431-432, Apr. 2007.

[4] P. V. Nikitin and K. V. S. Rao, "Theory and measurement of backscattering from RFID tags," IEEE Antennas Propagat. Mag., vol. 48, no. 6, pp. 212-218, Dec. 2006.

[5] C.-C. Yen, A. E. Gutierrez, D. Veeramani, and D. van der Weide, "Radar cross-section analysis of backscattering RFID tags," IEEE Antennas and Wireless Propagation Letters, vol. 6, pp. 279-281, 2007.

[6] F. Fuschini, C. Piersanti, F. Paolazzi, and G. Falciasecca, "Analytical approach to the backscattering from UHF RFID transpoder," IEEE Antennas and Wireless Propagation Letters, vol. 7, pp. 33-35, 2008.

[7] R. C. Hansen, "Relationships between antennas as scatterers and as radiators," Proc. IEEE, vol. 77, no. 5, pp. 659-662, May 1989.

[8] D. Tse and P. Viswanath, Fundamentals of Wireless Communication. Cambridge University Press, 2005.

[9] J. G. Proakis, Digital Communications, 4th ed. New York, NY, 10020: McGraw-Hill, Inc., 2001. 